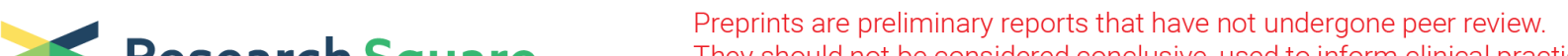

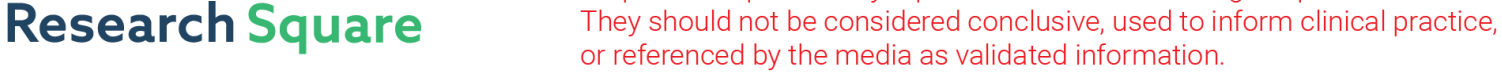 \\ Detection of anti-SARS-CoV-2 antibodies in patients with rheumatoid arthritis.
}

\section{Shomi Oka}

National Hospital Organization Tokyo National Hospital

\section{Takashi Higuchi}

National Hospital Organization Tokyo National Hospital

Hiroshi Furukawa ( $\nabla$ furukawa-tky@umin.org )

National Hospital Organization Tokyo National Hospital

\section{Kota Shimada}

National Hospital Organization Sagamihara National Hospital

\section{Atsushi Hashimoto}

National Hospital Organization Sagamihara National Hospital

\section{Toshihiro Matsui}

National Hospital Organization Sagamihara National Hospital

\section{Shigeto Tohma}

National Hospital Organization Tokyo National Hospital

\section{Short Report}

Keywords: anti-SARS-CoV-2 antibody, rheumatoid arthritis, rheumatoid factor, electrochemiluminescence immunoassay (ECLIA), immunochromatographic assay (ICA)

Posted Date: November 20th, 2020

DOl: https://doi.org/10.21203/rs.3.rs-112294/v1

License: (c) (1) This work is licensed under a Creative Commons Attribution 4.0 International License.

Read Full License 


\section{Detection of anti-SARS-CoV-2 antibodies in patients with rheumatoid arthritis.}

Shomi Oka ${ }^{1,2}$, Takashi Higuchi ${ }^{1,3}$, Hiroshi Furukawa ${ }^{1,2}$, Kota Shimada ${ }^{4,5}$, Atsushi Hashimoto $^{4,6}$, Toshihiro Matsui ${ }^{2,4}$, and Shigeto Tohma ${ }^{1,2}$.

${ }^{1}$ Department of Rheumatology, National Hospital Organization Tokyo National Hospital, 3-1-1 Takeoka, Kiyose, 204-8585, Japan. ${ }^{2}$ Clinical Research Center for Allergy and Rheumatology, National Hospital Organization Sagamihara National Hospital, 18-1 Sakuradai, Minami-ku, Sagamihara, 252-0392, Japan. ${ }^{3}$ Department of Nephrology, Ushiku Aiwa General Hospital, 896 Shishiko-cho, Ushiku, 300-1296 Japan. ${ }^{4}$ Department of Rheumatology, National Hospital Organization Sagamihara National Hospital, 18-1 Sakuradai, Minami-ku, Sagamihara, 252-0392, Japan. ${ }^{5}$ Department of Rheumatic Diseases, Tokyo Metropolitan Tama Medical Center, 2-8-29 Musashi-dai, Fuchu, 183-8524, Japan. ${ }^{6}$ Department of Internal Medicine, Sagami Seikyou Hospital, 6-2-11 Sagamiohno, Minami-ku, Sagamihara, 252-0303, Japan.

Address correspondence to Hiroshi Furukawa, MD, PhD: Department of Rheumatology, National Hospital Organization Tokyo National Hospital, 3-1-1 Takeoka, Kiyose, 204-8585, Japan. Phone: +81-42-491-2111, FAX: +81-42-494-2168, e-mail: furukawa-tky@umin.org 
Key words: anti-SARS-CoV-2 antibody, rheumatoid arthritis, rheumatoid factor, electrochemiluminescence immunoassay (ECLIA), immunochromatographic assay (ICA).

Running title: Anti-SARS-CoV-2 Ab in RA

Key points: $\operatorname{IgM}$ and $\operatorname{IgG}$ anti-severe acute respiratory syndrome coronavirus 2 (SARS-CoV-2) antibodies were detected by the immunochromatographic assay (ICA) in the serum samples collected from rheumatoid arthritis (RA) patients before and after the outbreak of coronavirus disease 2019 (COVID-19).

The levels of IgM and IgG rheumatoid factor were higher in RA patients with IgM anti-SARS-CoV-2 antibodies by ICA.

The detection of IgM anti-SARS-CoV-2 antibody by ICA was influenced by IgM or IgG rheumatoid factors in RA patients. 


\begin{abstract}
Objectives: The severe acute respiratory syndrome coronavirus 2 (SARS-CoV-2) causes coronavirus disease 2019 (COVID-19) and the outbreak of COVID-19 was reported in December 2019 in Wuhan, China. Serological test is conducted to discriminate prior infection of SARS-CoV-2. The influence of auto-antibodies on the results of anti-SARS-CoV-2 antibodies was investigated in a few studies. Here, we investigated whether the results of anti-SARS-CoV-2 antibodies would be modified in patients with rheumatoid arthritis (RA).
\end{abstract}

Methods: Patients with RA were recruited at Sagamihara National Hospital from July 2014 to October 2015 ( $\mathrm{n}=38,2014$ cohort) and at Tokyo National Hospital from June to October 2020 ( $n=93,2020$ cohort). Anti-SARS-CoV-2 antibodies were measured in collected sera from these RA patients by electrochemiluminescence immunoassay (ECLIA) or immunochromatographic assay (ICA).

Results: Anti-SARS-CoV-2 antibodies were not detected in all the samples form RA patients in both cohorts by the ECLIA. However, anti-SARS-CoV-2 antibodies were detected in the serum samples from three $(7.9 \%)$ in 2014 cohort by the ICA and fifteen $(16.1 \%)$ in 2020 cohort. The IgM rheumatoid factor levels were increased in RA patients with IgM anti-SARS-CoV-2 antibodies by ICA compared with RA without any 
anti-SARS-CoV-2 antibodies (mean \pm standard deviation [IU/ml], $1223.0 \pm 1308.7$ vs.

$503.6 \pm 1947.2, P=0.0101)$. The levels of IgG rheumatoid factor were also upregulated in RA patients with IgM anti-SARS-CoV-2 antibodies by ICA ( $4.0 \pm 0.7$ vs. $2.4 \pm 0.9$, $P=0.0013)$.

Conclusion: The results of IgM anti-SARS-CoV-2 antibody by the ICA would be modified by IgM or IgG rheumatoid factors in RA patients. 


\section{Introduction}

The severe acute respiratory syndrome coronavirus 2 (SARS-CoV-2) causes coronavirus disease 2019 (COVID-19) and the outbreak of COVID-19 was reported in December 2019 in Wuhan, China [1]. In the diagnosis of COVID-19, quantitative reverse transcription polymerase chain reaction for SARS-CoV-2 was performed from nasal swab, saliva, or sputum samples. On the other hand, serological test was conducted to discriminate prior infection of SARS-CoV-2. The cross reactivity of anti-SARS-CoV-2 antibodies with antibodies against SARS-CoV was reported [2]. The cross reactivity of auto-antibodies in autoimmune disease patients with antibodies against SARS-CoV-2 was reported in previous studies [3,4], but not in other studies [5-7]. Additionally, it was still unknown how long antibodies persisted after infection and if antibodies were protective against SARS-CoV-2. Hence, we investigated whether the results of serological tests of anti-SARS-CoV-2 antibodies would be influenced in patients with rheumatoid arthritis (RA).

\section{Materials and methods}

Patients and sera

Japanese patients with RA were recruited at Sagamihara National Hospital from July 
2014 to October 2015 ( $\mathrm{n}=38,2014$ cohort) and at Tokyo National Hospital from June to October 2020 ( $n=93,2020$ cohort). The RA patents fulfilled American College of Rheumatology criteria for RA [8] or Rheumatoid Arthritis Classification Criteria [9] and had not been diagnosed as COVID-19 before the collection of sera. Sera from the RA patients were collected and analyzed for anti-SARS-CoV-2 antibody. The study was reviewed and approved by The Research Ethics Committee of Tokyo National Hospital and Sagamihara National Hospital. Written informed consent was obtained from all the patients. This study was conducted in accordance with the principles expressed in the Declaration of Helsinki.

Anti-SARS-CoV-2 antibody analyses

$\operatorname{IgM}$ and IgG classes of anti-SARS-CoV-2 antibodies were detected using the electrochemiluminescence immunoassay (ECLIA) system (Elecsys Anti-SARS-CoV-2, Roche Diagnostics, Mannheim, Germany), according to the manufacturer's instruction. Briefly, $12 \mu \mathrm{l}$ of serum was incubated with recombinant nucleocapsid $(\mathrm{N})$ antigens labeled with Tris(2,2'-bipyridyl)ruthenium(II)-complexes or biotins. After addition of streptavidin-coated microparticles, the microparticles are magnetically captured on the electrode and induced-chemiluminescent emission is measured with cobas 8000 
analyzer with e801 module (Roche Diagnostics). The obtained data were analyzed and cut off indices (COI) were calculated. Samples with COI less than 1.0 were designated to be negative for anti-SARS-CoV-2 antibodies and samples with COI equal to or more than 1.0 positive. Anti-SARS-CoV-2 antibodies were also detected using immunochromatographic assay (ICA, GenBody COVID-19 IgM/IgG, GenBody, Cheonan, Korea), according to the manufacturer's instruction with slight modifications. Briefly, $100 \mu \mathrm{l}$ of serum was added to the sample port. After addition of 3 drops (approximate volume: $100 \mu \mathrm{l}$ ) of buffer to the same sample port, the results were interpreted in 1 hour. The presence of only one band in the control line indicates a negative result; the presence of two bands in the control line and $\operatorname{IgM}$ or $\operatorname{IgG}$ test line indicates a positive result for IgM or IgG, respectively. Rheumatoid factor was detected by N-latex RF kit (Siemens Healthcare Diagnostics, München, Germany) or N-Assay LA RF-K Nittobo (Nittobo Medical, Koriyama, Japan) and IgG rheumatoid factor was measured by Smitest IgG RF ELISA (Medical \& Biological Laboratories, Nagoya, Japan). Anti-citrullinated peptide antibody was measured by Mesacup-2 test CCP (Medical \& Biological Laboratories). 
Presence of anti-SARS-CoV-2 antibody was analyzed by Fisher's exact test using 2X2 contingency tables. Differences of demographic features of RA patients were analyzed by Fisher's exact test using 2X2 contingency tables or Mann-Whitney's U test. Statistical significance was defined as $P<0.05$.

\section{Results}

Anti-SARS-CoV-2 antibody profiles were analyzed in 2014 and 2020 cohorts. Anti-SARS-CoV-2 antibodies were not detected in all the samples form RA patients in both cohorts by the ECLIA. However, the ICA predicted that serum samples from three (7.9\%) of RA patients were positive for anti-SARS-CoV-2 antibodies in 2014 cohort and fifteen (16.1\%) in 2020 (Table 1). The positive rates of anti-SARS-CoV-2 antibodies were not significantly different between these two cohorts $(P=0.2720)$. Thus, anti-SARS-CoV-2 antibodies were not detected in all the RA patients by the ECLIA, though some samples of both cohorts were predicted to be positive by the ICA.

Characteristics of the RA with or without reactivity to the ICA were described in Table 2. Rheumatoid factor levels were increased in RA patients with IgM anti-SARSCoV-2 antibodies by ICA compared with RA without any anti-SARS-CoV-2 antibodies (mean \pm standard deviation $[\mathrm{IU} / \mathrm{ml}], 1223.0 \pm 1308.7$ vs. $503.6 \pm 1947.2, P=0.0101)$. The 
levels of IgG rheumatoid factor were also upregulated in RA patients with IgM anti-SARS-CoV- 2 antibodies by ICA ( $4.0 \pm 0.7$ vs. $2.4 \pm 0.9, P=0.0013)$. Rheumatoid factor levels were comparable in RA patients with IgG anti-SARS-CoV-2 antibodies by ICA. The levels of IgG rheumatoid factor were not different in RA patients with IgG anti-SARS-CoV-2 antibodies by ICA. There were no differences of mean age, percentage of males, or anti-citrullinated peptide antibodies. Thus, the results of IgM anti-SARS-CoV-2 antibodies by ICA was influenced in RA patients with rheumatoid factors.

\section{Discussion}

Although anti-SARS-CoV-2 antibodies was not detected by the ECLIA in all the RA samples, some samples were predicted to be positive by the ICA in the two cohorts. The positive rates of anti-SARS-CoV-2 antibodies by the ICA were comparative between these cohorts. Since samples from 2014 cohort were collected before the outbreak of COVID-19, anti-SARS-CoV-2 antibodies could not be detected in the cohort, indicating the false positive reactions in the sera from these RA patients. In the present study, rheumatoid factors were detected by latex fixation tests that mainly measured the IgM class of rheumatoid factor. Because levels of rheumatoid factor were higher in the RA 
patients with reactivity to the ICA, it was suggested that IgM rheumatoid factor could react with the anti-human IgM antibodies in the ICA kit to cause the false positive reaction. It was also suggested that $\operatorname{IgG}$ rheumatoid factor reacted with the anti-human IgM antibodies in the ICA kit. However, the results of IgG anti-SARS-CoV-2 antibodies by the ICA were not influenced by IgM or IgG rheumatoid factors. These results suggested that both of $\operatorname{IgM}$ and $\operatorname{IgG}$ rheumatoid factor might not react with anti-human IgG antibodies in the ICA kit and that other factors would modify the results of IgG anti-SARS-CoV-2 antibodies by the ICA in RA patients [10]. The interference of IgM rheumatoid factor with immunological assays was reported in previous studies [11-14], including those on anti-SARS-CoV-2 antibody assays [4], though the effects of IgG rheumatoid factor was poorly investigated. It is still unknown which of $\operatorname{IgM}$ or $\operatorname{IgG}$ rheumatoid factors predominantly modified the results of IgM anti-SARS-CoV-2 antibodies by the ICA. Thus, the results of IgM anti-SARS-CoV-2 antibodies by the ICA would be modified by IgM or IgG rheumatoid factors in RA patients, but those of IgG by other factors.

To the best of our knowledge, this is the first analysis on the effects of IgG rheumatoid factors on the results by the the ICA for SARS-CoV-2 in RA patients. The results by the ICA should be validated by the ECLIA or other methods, when anti-SARS-CoV-2 
antibodies are measured in RA patients. Since the sample size of the present study is modest, larger scale independent studies should be performed to validate these results from this study. Antibody profiles in patients with other autoimmune diseases than RA were not analyzed in the present study; anti-SARS-CoV-2 antibodies should be measured by the ICA and ECLIA in other autoimmune disease patients in future studies. It should be clarified what modified the results of IgG anti-SARS-CoV-2 antibodies by the ICA in RA patients.

\section{Funding}

The work was supported by Grants-in-Aid for Clinical Research from National Hospital Organization, and research grants from the following pharmaceutical companies: Bristol-Myers Squibb Co., Abbott Japan Co., Ltd., Astellas Pharma Inc., Chugai Pharmaceutical Co., Ltd., Eisai Co., Ltd., Mitsuibishi Tanabe Pharma Corporation, Merck Sharp and Dohme Inc., Pfizer Japan Inc., Takeda Pharmaceutical Company Limited, Teijin Pharma Limited. The funders had no role in study design, data collection and analysis, decision to publish, or preparing the manuscript.

\section{Contributors}


Conceived and designed the experiments: HF and ST, Performed the experiments: SO, TH, and HF, Analyzed the data: HF and ST, Contributed reagents/materials/analysis tools: HF, KS, AH, TM, and ST, Wrote the manuscript: SO, HF, and ST.

\section{Conflict of interest statement}

HF was supported by research grants from Bristol-Myers Squibb Co. HF received honoraria from Ajinomoto Co., Inc., Daiichi Sankyo Co., Ltd., Dainippon Sumitomo Pharma Co., Ltd., Pfizer Japan Inc., and Takeda Pharmaceutical Company. ST was supported by research grants from 9 pharmaceutical companies: Abbott Japan Co., Ltd., Astellas Pharma Inc., Chugai Pharmaceutical Co., Ltd., Eisai Co., Ltd., Mitsubishi Tanabe Pharma Corporation, Merck Sharp and Dohme Inc., Pfizer Japan Inc., Takeda Pharmaceutical Company Limited, Teijin Pharma Limited. ST received honoraria from Asahi Kasei Pharma Corporation, Astellas Pharma Inc., AbbVie GK., Chugai Pharmaceutical Co., Ltd., Ono Pharmaceutical Co., Ltd., Mitsubishi Tanabe Pharma Corporation, Pfizer Japan Inc. The other authors declare no financial or commercial conflict of interest. 


\section{References}

1. Huang C, Wang Y, Li X, Ren L, Zhao J, Hu Y, Zhang L, Fan G, Xu J, Gu X, Cheng Z,

Yu T, Xia J, Wei Y, Wu W, Xie X, Yin W, Li H, Liu M, Xiao Y, Gao H, Guo L, Xie J, Wang G, Jiang R, Gao Z, Jin Q, Wang J, Cao B (2020) Clinical features of patients infected with 2019 novel coronavirus in Wuhan, China. Lancet 395 (10223):497-506 2. Zhou P, Yang XL, Wang XG, Hu B, Zhang L, Zhang W, Si HR, Zhu Y, Li B, Huang CL, Chen HD, Chen J, Luo Y, Guo H, Jiang RD, Liu MQ, Chen Y, Shen XR, Wang X, Zheng XS, Zhao K, Chen QJ, Deng F, Liu LL, Yan B, Zhan FX, Wang YY, Xiao GF, Shi ZL (2020) A pneumonia outbreak associated with a new coronavirus of probable bat origin. Nature 579 (7798):270-273

3. Paiva KJ, Grisson RD, Chan PA, Huard RC, Caliendo AM, Lonks JR, King E, Tang EW, Pytel-Parenteau DL, Nam GH, Yakirevich E, Lu S (2020) Validation and performance comparison of three SARS-CoV-2 antibody assays. J Med Virol 2020 $(25): 26341$

4. Wang Q, Du Q, Guo B, Mu D, Lu X, Ma Q, Guo Y, Fang L, Zhang B, Zhang G, Guo X (2020) A Method To Prevent SARS-CoV-2 IgM False Positives in Gold Immunochromatography and Enzyme-Linked Immunosorbent Assays. J Clin Microbiol 58 (6):00375-00320 
5. Teng J, Dai J, Su Y, Zhou Z, Chi H, Wan L, Meng J, Wang Z, Wang F, Ma Y, Hu Q,

Cheng X, Liu H, Ye J, Shi H, Sun Y, Yang C, Wang X (2020 ) Detection of IgM and IgG antibodies against SARS-CoV-2 in patients with autoimmune diseases. Lancet

Rheumatol 2 ((7)):e384-e385.

6. Manalac J, Yee J, Calayag K, Nguyen L, Patel PM, Zhou D, Shi RZ (2020)

Evaluation of Abbott anti-SARS-CoV-2 CMIA IgG and Euroimmun ELISA IgG/IgA

assays in a clinical lab. Clin Chim Acta 510:687-690

7. Lau CS, Hoo SP, Yew SF, Ong SK, Lum LT, Heng PY, Tan JG, Wong MS, Aw TC

(2020) Evaluation of an Electrochemiluminescent SARS-CoV-2 Antibody Assay. J Appl

Lab Med 5 (6):1313-1323

8. Arnett FC, Edworthy SM, Bloch DA, McShane DJ, Fries JF, Cooper NS, Healey LA, Kaplan SR, Liang MH, Luthra HS, et al. (1988) The American Rheumatism Association 1987 revised criteria for the classification of rheumatoid arthritis. Arthritis Rheum 31

(3):315-324.

9. Aletaha D, Neogi T, Silman AJ, Funovits J, Felson DT, Bingham CO, 3rd, Birnbaum NS, Burmester GR, Bykerk VP, Cohen MD, Combe B, Costenbader KH, Dougados M, Emery P, Ferraccioli G, Hazes JM, Hobbs K, Huizinga TW, Kavanaugh A, Kay J, Kvien TK, Laing T, Mease P, Menard HA, Moreland LW, Naden RL, Pincus T, Smolen JS, 
Stanislawska-Biernat E, Symmons D, Tak PP, Upchurch KS, Vencovsky J, Wolfe F, Hawker G (2010) 2010 Rheumatoid arthritis classification criteria: an American College of Rheumatology/European League Against Rheumatism collaborative initiative. Arthritis Rheum 62 (9):2569-2581

10. Degn SE, Andersen SH, Jensen L, Thiel S, Jensenius JC (2011) Assay interference caused by antibodies reacting with rat kappa light-chain in human sera. J Immunol Methods 372 (1-2):204-208

11. Kazantseva L, García Lázaro MDP, Herrera-Velit P, Espinoza JR (2017) Anti-Fas2 IgM antibodies in Fasciola hepatica infected patients with positive IgG serology. Trans R Soc Trop Med Hyg 111 (3):102-106

12. Kragstrup TW, Vorup-Jensen T, Deleuran B, Hvid M (2013) A simple set of validation steps identifies and removes false results in a sandwich enzyme-linked immunosorbent assay caused by anti-animal $\operatorname{IgG}$ antibodies in plasma from arthritis patients. Springerplus 2 (1):263

13. Malyak M, Joslin FG, Verderber EL, Eisenberg SP, Arend WP (1991) IL-1ra ELISA: reduction and alkylation of synovial fluid eliminates interference by $\operatorname{IgM}$ rheumatoid factors. J Immunol Methods 140 (2):281-288

14. Briantais MJ, Grangeot-Keros L, Pillot J (1984) Specificity and sensitivity of the 
IgM capture immunoassay: studies of possible factors inducing false positive or false negative results. J Virol Methods 9 (1):15-26 
Table 1. Positive rates of anti-SARS-CoV2 antibodies by ICA in the RA patients.

\begin{tabular}{llll}
\hline & 2014 cohort & 2020 cohort & $P$ \\
\hline Number & 38 & 93 & \\
Anti-SARS-CoV-2 Ab positive, $\mathrm{n}(\%)$ & $3(7.9)$ & $15(16.1)$ & 0.2720
\end{tabular}

SARS-CoV-2: severe acute respiratory syndrome coronavirus 2, ICA: immunochromatographic assay, RA: rheumatoid arthritis. Numbers of RA patients with IgM or IgG anti-SARS-CoV-2 antibodies are shown. Percentages are shown in parenthesis. Difference was tested by Fisher's exact test using $2 \mathrm{X} 2$ contingency tables. 
Table 2. Characteristics of RA patients with or without anti-SARS-CoV-2 antibodies detected by ICA.

\begin{tabular}{|c|c|c|c|c|c|c|c|}
\hline & $\operatorname{IgM}$ or $\operatorname{Ig} G(+)$ & $P$ & $\operatorname{IgM}(+)$ & $P$ & $\operatorname{IgG}(+)$ & $P$ & $\operatorname{IgM}$ or $\operatorname{IgG}(-)$ \\
\hline Number & 18 & & 5 & & 14 & & 113 \\
\hline Mean age, years (SD) & $74.8(10.2)$ & 0.1402 & $73.6(13.4)$ & 0.4072 & $75.8(9.2)$ & 0.1292 & $70.7(11.3)$ \\
\hline Male, n (\%) & $3(16.7)$ & $0.7630 *$ & $2(40.0)$ & $0.5953 *$ & $1(7.1)$ & $0.3018 *$ & $27(23.9)$ \\
\hline $\mathrm{RF}, \mathrm{IU} / \mathrm{ml}$ (SD) & $464.8(812.8)$ & 0.0607 & $1223.0(1308.7)$ & 0.0101 & $181.0(177.9)$ & 0.3739 & $503.6(1947.2)$ \\
\hline ACPA, IU/ml (SD) & $299.6(277.5)$ & 0.5099 & $219.7(233.5)$ & 0.6161 & $306.8(297.1)$ & 0.4857 & $340.3(659.1)$ \\
\hline IgG RF, IU/ml (SD) & $2.8(1.0)$ & 0.1101 & $4.0(0.7)$ & 0.0013 & $2.5(0.8)$ & 0.7348 & $2.4(0.9)$ \\
\hline
\end{tabular}

SARS-CoV-2: severe acute respiratory syndrome coronavirus 2, ICA: immunochromatographic assay, RA: rheumatoid arthritis, RF: rheumatoid factor, ACPA: anti-citrullinated peptide antibody, IgM or IgG $(+)$ : RA patients with IgM or IgG anti-SARS-CoV-2 antibodies, IgM (+):RA patients with IgM anti-SARS-CoV-2 antibodies, IgG (+): RA patients with IgG anti-SARS-CoV-2 antibodies, IgM or IgG (-): RA patients without IgM or IgG anti-SARS-CoV-2 antibodies. Number or Average values of each group are shown. Standard deviations or percentages are shown in parenthesis. Difference was tested in the comparison with IgM or IgG (-) group by Fisher's exact test using 2X2 contingency tables or Mann-Whitney's U test. *Fisher's exact test was employed. 